\title{
EL LENGUAJE ORAL COMO PUENTE ENTRE EL INGRESANTE Y LA DISGIPLINA PROYEGTUAL: UNA EXPERIENCIA EN LOS TALLERES DE DISEÑO ${ }^{1}$
}

\author{
ORAL LANGUAGE AS A BRIDGE BETWEEN THE \\ ENTRANT AND THE PROJEGTUAL DISCIPLINE. AN \\ EXPERIENGE IN THE DESIGN WORKSHOPS
}

\author{
Claudia Cutrera \\ Universidad Nacional de Mar del Plata - Argentina
}

Arquitecta y profesora argentina. Graduada de la Universidad Nacional de Mar del Plata, se especializó en Gestión y Planificación del Paisaje en la Universidad Nacional de La Plata. Es investigadora en temas de paisaje y pedagogía universitaria en el Instituto del Hábitat y del Ambiente (UNMdP) Participa en congresos nacionales e internacionales sobre temas pedagógicos y paisajísticos. Es Profesora Adjunta del Área Proyectual, docente de la asignatura Bienes Naturales y Paisajísticos en la Tecnicatura Universitaria en Gestión Cultural y participa en materias electivas sobre Paisaje y Espacio Público en la Facultad de Arquitectura, Urbanismo y Diseño de la UNMdP.

claudiacutrera@gmail.com

Fecha de recepción: 13 de abril, 2018 / Aceptación: 20 de mayo, 2018. 


\section{Resumen}

Podemos aseverar que pertenecer a un grupo, sea este cultural, disciplinar o etario, está definido, entre otras cosas, por compartir un lenguaje común. Este lenguaje oral es un instrumento de comunicación y, a la vez, es la herramienta que nos permite construir nuestros pensamientos y hace posible la generación de conocimiento. Las representaciones de dibujos y maquetas, en sus versiones digitales 0 analógicas, así como las fotografías, entre otras técnicas, son lenguajes específicos de las disciplinas proyectuales; a través de ellos pensamos y comunicamos nuestras ideas y pensamientos. Pero también

en el proceso de enseñanza del diseño es primordial la palabra para iniciar al estudiante en la comprensión, uso y gestión de esos lenguajes específicos que lo acercarán y habilitarán para el pensamiento proyectual. La enseñanza de un lenguaje específico y los códigos propios de la disciplina tienen una presencia permanente en los talleres proyectuales. Se produce igual fenómeno que con la disciplina misma, se aprende a partir de la práctica y de la reflexión sobre esa práctica. Si afirmamos que "a proyectar se aprende proyectando" podemos decir que "se aprende el lenguaje disciplinar argumentando sobre el proyecto y para argumentar se necesita la palabra (Cutrera, 2016b, p. 1),

esa palabra que connota significados propios de la disciplina. En este sentido indagaremos en la importancia del lenguaje oral como mediador entre lenguajes específicos de la disciplina proyectual y en el valor del silencio como espacio de reflexión. Cuanto más aprenda el estudiante el lenguaje específico de nuestra disciplina, mejor será su producción en el campo proyectual y mayor será su comprensión de la arquitectura.

\section{Palabras clave}

$$
\text { Didáctica-lenguaje-taller proyectual-diálogo-palabra- silencio-mediación. }
$$

\section{Abstract}

It is possible to affirm that belonging to a group, either cultural, disciplinary, or age group, is defined among other things by sharing a common language. This oral language is an instrument for communication as well as a tool that allows the construction of thoughts that enhance the generation of knowledge. The representations of drawings and models in their digital or analogue version, as well as photographs and other techniques, are specific languages of the project disciplines through which we think and communicate ideas and thoughts. Also, in the process of teaching design, the word is essential to initiate the student in the comprehension, use, and management of those specific languages that will approach and enable students for project thinking. The teaching of a specific language and the codes of the discipline have a permanent presence in project workshops. The same phenomenon occurs with the discipline itself which is learned from practice and reflection on practice. If we affirm that how "to project is learned by projecting" we can say that " we learn the disciplinary language by arguing about the project, and in order to argue, the word is needed" (Cutrera, 2016b, p.1), as the word connotes meanings typical to the discipline. In this sense we will investigate the importance of oral language as a mediator between specific languages of design and the value of silence as a space for reflection. The more the student learns the specific language of our discipline, the better their production and understanding is in the field of design and architecture.

\section{Keywords}

\section{Didactic-language-workshop project-dialogue-word-silence-mediation.}




\section{A modo de introducción}

Son las primeras clases en el taller de diseño... Estoy aquí porque tempranamente descubrí mi vocación para "hacer casas a la gente". Siendo adolescente repetía una y otra vez esta frase cuando me preguntaban que iba a estudiar y por qué... Necesito cortar el cartón para hacer una maqueta no tengo muchos datos y mucho menos cuento con habilidades para estas tareas, admiro a mis compañeros que vienen de la escuela técnica y trabajan afanosamente y con orgullo al lado de nosotros, los bachilleres. De golpe siento un fuerte dolor en mi pierna, la trincheta atravesó el cartón y llegó a mi ahora tajeado pantalón. El dolor, muy agudo, pronto se convirtió en vergüenza. Con la mayor premura posible salgo fuera del taller para curarme la herida. Al salir me cruzo con el docente que me pregunta preocupado, ¿qué te pasó?, le respondo con rapidez y mucho calor en la cara: me corté con el escalímetro (Cutrera, 2016b, p. 1).

Esta anécdota que una y otra vez nos relata una colega ya recibida hace algunos años, demuestra, en pocas palabras, las dificultades que afrontamos los que nos iniciamos en esta aventura de aprender a proyectar. Quizás no se conozcan algunos términos utilizados en el relato; para quienes no estén familiarizados con están prácticas, podemos describir al escalímetro como un tipo de regla que permite medir los objetos, dibujos o maquetas en diferentes escalas de acuerdo a la actividad o requerimiento de los ejercicios de taller; en cuanto a la trincheta, actualmente más conocida como cutter, es un objeto con filo que se utiliza para cortar distintos materiales.

La narración que presentamos es muy elocuente y demuestra con claridad la intención de revelar el valor de la palabra como mediadora en el proceso de enseñanza y aprendizaje. Nos ejemplifica de modo sintético los inconvenientes que afrontan los estudiantes en los primeros meses de nuestras carreras y las acciones que los docentes realizamos para introducir al ingresante en el campo de las disciplinas proyectuales.

Indagaremos sobre los aspectos de la comunicación entre los actores que participan de los talleres de las asignaturas proyectuales. Toda disciplina u oficio posee sus propios códigos que deben ser aprendidos por quienes deseen o necesiten desarrollar esa actividad. En los talleres proyectuales también existe un lenguaje verbal disciplinar, común a todos, que permite la comunicación e interpretación de conceptos y mensajes que, tanto estudiantes como docentes, se trasmiten unos a otros, aparte de los lenguajes de representación propios de la disciplina; asimismo, el lenguaje proyectual hace uso de la palabra en la producción del proyecto. Cabe señalar que, a diferencia de estos lenguajes disciplinares específicos, el lenguaje verbal está vinculado a nuestra lengua materna y como tal nos acompaña desde nuestra primera infancia. Por este motivo es tan significativo que sea también el primer vínculo que nos enlaza a estudiantes, docentes y objetos o hechos arquitectónicos proyectados.

A los efectos de esta publicación no profundizaremos en distintas corrientes que versan sobre los estudios del lenguaje y la lengua materna; aunque nos mueve a una reflexión ¿Se aprende el lenguaje de la arquitectura igual que se aprende el lenguaje materno? Los conductistas sostienen que el lenguaje se adquiere a partir de "condicionamiento operante", mediante el proceso de estímulo-respuesta-recompensa. Los bebés hacen sonidos, que si son parecidos a las palabras son recompensados por los padres, y de a poco se va estableciendo una relación, por reiteración, entre la palabra y el objeto que representa. ¿Sucede igual con conceptos más abstractos? ¿Comprende el estudiante conceptos como "la fluidez del espacio moderno" o "la condición estática del espacio clásico" luego que el docente se lo señale muchas veces bajo este nombre? En cambio, si tomamos las teorías innatistas de Chomsky, no parece que pudiera aplicarse tan directamente al campo arquitectónico, ya que no solamente habla- 
mos de la condición de la percepción de un espacio, sino de la construcción de herramientas operativas para trabajar sobre ese espacio, habilidades que no son innatas a los humanos, sino que tiene que ver con saberes adquiridos de aquellos que estudian la disciplina arquitectónica.

Quizás el constructivismo social, de la mano de Vigotsky y Bruner, sea la que se acerca más al uso de la palabra y los modos de construir el conocimiento en los talleres proyectuales. Vigotsky es uno de los primeros en sostener que el habla es fundamental para la construcción de los procesos mentales, señala que a través del habla se desarrolla una doble función, la comunicación externa con los otros y la interna con los pensamientos. Algo parecido sucede en los diálogos que se desarrollan en los talleres proyectuales, entre los actores y con los proyectos.

Como docentes de asignaturas proyectuales empleamos modos tanto explícitos como implícitos para comunicarnos con nuestros estudiantes. El objetivo de este espacio

es reflexionar sobre el lenguaje oral específico de la disciplina, para luego explorar más allá del mismo, (...) ¿Qué necesita escuchar el estudiante para avanzar en su proceso de aprendizaje a través del proceso proyectual?, ¿cómo comunica el docente la corrección para que se produzca conocimiento en el estudiante? (Cutrera, 2016b, p. 2).

En este sentido entendemos que no basta con aprender el significado de cada palabra sino también en qué momento y circunstancia se emplea. lenguaje el

Consideramos como parte esencial del

valor de los silencios, tan importantes como las palabras mismas. Tanto docente como estudiante también deben aprender a "escuchar" los silencios, los propios y los del otro, en esos momentos tienen lugar la reflexión y la asimilación de lo transmitido, de la recepción y la emisión de los mensajes (Cutrera, 2016b, p. 2).

Seguramente coincidimos en que, en cualquier proceso de aprendizaje, el conocimiento se produce en el estudiante y en el proceso proyectual se verifica su proceso de aprendizaje. Durante las correcciones en los talleres proyectuales, los docentes mostramos caminos y ayudamos al estudiante para que reflexione sobre sus acciones, fundamentalmente a través del lenguaje oral (Cutrera, 2016b, p. 2).

\section{Sobre las asignaturas proyectuales}

Previo al desarrollo del tema, sentimos el compromiso de citar algunas de las cuestiones que definen a la acción de proyectar, y particularmente a aquellas que corresponden al aprendizaje de estas acciones. Los estudiantes de las disciplinas proyectuales aprenden desde la acción misma de proyectar, referiremos esta afirmación a los conceptos de Donald Schön (2010) "La paradoja de aprender una competencia realmente nueva es la siguiente: que un estudiante no puede, al principio, comprender lo que necesita aprender, solo puede aprenderlo formándose a sí mismo comenzando por hacer lo que aún no comprende" (p. 93).

Para comenzar diremos que en la enseñanza de las asignaturas proyectuales las respuestas a una problemática planteada son múltiples y cada una de ellas está validada por las intenciones y propuestas ideadas por el estudiante. Es la diferencia fundamental con otras disciplinas en las cuales para cada problema hay solo una o a lo sumo dos respuestas correctas, 0 aquellas disciplinas en las que el aprendizaje se verifica con la asimilación de conceptos y contenidos estudiados 0 transmitidos previamente por los expertos. Esta particularidad, donde cada proyecto es ideado y desarrollado por cada estudiante, partiendo todos desde el mismo programa e incluso a veces en un mismo emplazamiento y que da la posibilidad de tantas respuestas como alumnos haya, resulta productiva para el proceso de aprendizaje de los estudiantes y también colabora con el desarrollo de la tarea pedagógica del docente de diseño. Así es como, cuanta más varie- 
dad de propuestas se generen, más posibilidades de aprendizaje sobre las problemáticas que se plantean en cada ejercicio.

En la didáctica de taller valoramos los beneficios de trabajar en equipo, sea este entre estudiantes, por comisiones o el taller total; el trabajo generado con esta dinámica favorece los procesos de enseñanza y aprendizaje. Se origina una producción colectiva en la cual lo que elabora cada parte enriquece al aprendizaje del otro, y el intercambio de pareceres genera nuevas soluciones. Estas reflexiones nos dejan comprender algo más de las dinámicas de los procesos pedagógicos que se producen en el taller proyectual, con estos conceptos iniciamos el tema que nos concierne, el aprendizaje y uso del lenguaje oral disciplinar en el primer año de las carreras proyectuales.

\section{Del lenguaje oral al lenguaje proyectual}

Tanto en las más tradicionales formas de considerar la comunicación como en aquellas más modernas, es imprescindible disponer de un código común a ambas partes para que se efectivice dicha comunicación. Este hecho motiva la necesidad de hacer consciente en el estudiante e informarlo sobre la existencia de esos códigos, propios de la disciplina, para que comprenda los mensajes que se materializarán en correcciones, críticas y diálogos sobre el proyecto, garantizando la comunicación necesaria para que se produzca conocimiento (Cutrera, 2016b, p. 3),

es decir, para que el estudiante aprenda.

En este sentido nos preguntamos de qué manera se produce el aprendizaje de dichos códigos en el taller proyectual. En este espacio pedagógico el docente enseña términos vinculados a la disciplina proyectual, pero a su vez debe homologarlos con términos de uso más cotidiano y familiar que reconozca el alumno; así en esta constante comparación y descripción el estudiante va incorporando palabras que pertenecen al lenguaje disciplinar y de ese modo va aprendiendo términos y significados propios de la asignatura. Esta transición que mencionamos y que está directamente relacionada con el uso de la palabra, se realiza durante el proceso de enseñanza de la disciplina misma. Por lo que resulta difícil separar el proceso de aprendizaje de cada asignatura con el del aprendizaje del lenguaje específico de la disciplina. En la medida que más aprendamos un lenguaje y comprendamos sus códigos, mejorará nuestro conocimiento sobre el tema y los nuevos conceptos nutrirán nuestros pensamientos para entablar mejores relaciones en la producción proyectual.

Las primeras clases nos resulta fácil explicar a los estudiantes el significado 0 aspecto de los objetos, como por ejemplo un escalímetro 0 una trincheta, que están al alcance de nuestras manos para mostrarlos describiendo características y usos. En cambio, cuando hablamos de conceptos más abstractos, y con los cuales no están familiarizados, la comprensión no es tan directa y efectiva, nos referimos más específicamente a cómo comunicar al estudiante qué es proyectar. En la experiencia de nuestra cátedra ${ }^{2}$ iniciamos la cursada con trabajos prácticos breves vinculados con ejemplos de otras artes que pertenecen a nuestra cultura cuyo objetivo es analizar y observar hechos culturales conocidos por los estudiantes. En los siguientes ejercicios, introducimos a los estudiantes en la observación y análisis de hechos arquitectónicos. En la dinámica de estas prácticas preliminares comienza el aprendizaje de códigos específicos de la disciplina y los modos de comunicarnos a través del lenguaje oral. Paulatinamente nos acercamos a conceptos del campo disciplinar y al manejo de un lenguaje específico, que nos permitirá "hablar" sobre el proyecto y, sobre todo, proyectar o pensar en el proyecto. Estaremos iniciando a nuestros estudiantes en el lenguaje proyectual.

En nuestra práctica docente comenzamos utilizando un lenguaje cuyos significados van de lo más general y conocido a otros significados más específicos y propios de la disciplina. Visualizamos conceptos a través 
de información gráfica y en maquetas, de modo de ir aprendiendo el léxico propio de la asignatura; luego, a partir de ejercicios experimentales y cortos los estudiantes comienzan a hacer, es decir, comienzan a proyectar. La paradoja es que una vez que el ejercicio está hecho, los estudiantes reconocen que estuvieron proyectando, casi sin darse cuenta (Cutrera, 2016 b, p. 4); utilizaron nuevos códigos de comunicación y determinados lenguajes, compartieron la producción con sus compañeros, comenzaron a entender los mecanismos de trabajo de las didácticas proyectuales en el espacio pedagógico del taller proyectual.

A través de los primeros ejercicios, docentes y estudiantes buscan establecer acuerdos y ambas partes comienzan unos a dar a conocer y otros a construir los códigos que les servirán para el resto de su carrera y vida profesional. Se presenta a los estudiantes un lenguaje común, propios del ámbito de taller y de la disciplina, se incorporan definiciones del campo de lo proyectual y se especifican los términos y sus significados. En la medida que se ejercita el uso de un nuevo léxico, se demuestra la necesidad del mismo para completar los otros lenguajes utilizados para proyectar, como el dibujo a mano alzada, la modelización en maqueta (analógica o digital), el dibujo técnico, la fotografía, entre otros (Cutrera, 2016b, p. 4).

El estudiante que ingresa es novato en la práctica y uso de estos lenguajes disciplinares, por eso es tan importante el uso de la palabra como mediadora entre lo que piensa y aquello que desarrolla en sus proyectos e ideas. En las asignaturas proyectuales generar diálogos es fundamental para los procesos de enseñanza y de aprendizaje; en el taller como ámbito pedagógico se originan distintos pares dialógicos entre sus actores, es decir entre docente y estudiante, entre estudiante y estudiante, entre docente y docente $y$, entre estudiante y proyecto. Estos pares de diálogo y de trabajo se entrelazan y relacionan; no podrían existir unos sin los otros, y su importancia radica en que nos permiten generar conocimiento. Tomando los conceptos de Mazzeo y Romano, "el diálogo en la enseñanza está lejos de ser una mera conversación amistosa, tiene una función didáctica, exploratoria, interrogativa, intencionalmente planificada y con objetivos claros" (Mazzeo, Romano, 2007, p. 123).

Son muchos los modos en que utilizamos el lenguaje oral. Por medio de la palabra el estudiante comunica y argumenta sus proyectos, ideas y dudas, cómo avanza y qué falta resolver y, con la ayuda de nuestra pregunta, puede desarrollar el proceso de sus pensamientos. El docente, por su parte, retoma con la palabra situaciones descriptas por el estudiante que se visualizan en el proyecto para realizar correcciones y críticas, para clarificar conceptos e indicar posibles caminos.

Desde la experiencia los docentes sabemos la importancia del buen uso del lenguaje oral: en qué momento se expresa cada concepto, cómo se transmite, cuáles son los más pertinentes en relación al momento del proceso de aprendizaje y del proyecto, diferenciar la necesidad de una corrección o de una crítica, y estas son algunas de las acciones que ineludiblemente están vinculadas al uso de la palabra. Además de este discurso unidireccional del docente al estudiante, consideramos fundamental la generación de un diálogo entre estos actores, y en esta "conversación" destacamos el rol de la pregunta.

"Durante el desarrollo del proyecto el docente realiza preguntas para ser respondidas en la corrección y también para demostrar la importancia de las mismas en todo proceso proyectual" (Cutrera, 2016a, p. 195). Está en el docente conducir el aprendizaje a través de la pregunta y favorecer la evolución del proyecto y colaborar con la comprensión del estudiante. No solo preguntar al estudiante, sino también preguntar al proyecto, lo que Gregotti llama la conversación proyectual.

Cuando el estudiante aprende a preguntar y repreguntar al proyecto, reconoce que es imprescindible la reflexión durante el proceso de aprendizaje y como docentes garan- 
tizamos que se incorporen las preguntas y el diálogo con el proyecto como parte del método para proyectar, para iniciarse en su vida académica y para futuros procesos proyectuales que abordará como estudiante o como profesional. De este modo no solo logramos que el estudiante sea consciente que está proyectando sino, sobre todo, que está aprendiendo a proyectar (Cutrera, 2016b, p. 4).

\section{El diálogo: conversaciones sobre y con el proyecto}

Los seres humanos vivimos gracias a la comunicación, y muchas de las prácticas que consideramos que nos definen como seres humanos son el resultado directo de la manera en que nos comunicamos: el lenguaje, el razonamiento, la moralidad y la organización social (Burbules, 1999, p. 35).

Esta cita expresa cómo el diálogo es inherente a la condición humana, traslademos esta reflexión al campo de las disciplinas proyectuales. Ya expresamos que en toda comunicación se necesita un lenguaje común a las partes para que se entiendan. Si nos referimos al caso particular de la enseñanza en los talleres de diseño, además de la transmisión de docente a estudiante de determinados significados o conceptos, se produce una interrelación producto de los diálogos que se generan entre los pares docente/estudiante y estudiante/ proyecto. Estas conversaciones, donde además intervienen otros lenguajes propios de la disciplina, se desarrollan en el marco de una didáctica proyectual, donde es difícil de separar lo que se comunica con lo que comunica el proyecto. En la medida que interpretan el mensaje del otro y reconocen sus significados, tanto estudiantes como docentes aprenden que estos varían de acuerdo al momento del proceso proyectual. Una vez interpretadas las intenciones del estudiante o las correcciones del docente, cada parte reflexiona y elabora una respuesta didáctica, el docente y una propuesta proyectual el estudiante, de acuerdo a la problemática planteada y los obje- tivos pedagógicos de la asignatura, así se produce aquello que D. Schön (2010) Ilama "convergencia de significados", es decir entre ambas partes construyen conocimiento, el docente desde lo pedagógico y el estudiante desde lo proyectual.

Por otro lado, y completando esta relación dialógica entre docente, estudiante y proyecto, destacamos la importancia del entorno donde se desarrollan estos acuerdos de significados y lenguajes. "Nos referimos a la metodología participativa del taller como ámbito de generación de conocimiento y como contexto físico y pedagógico" (Cutrera, 2016b, p. 5) y especialmente como el lugar donde los estudiantes realizan su proceso proyectual. Otra cuestión que consideramos relevante

son las acciones que acompañan a las palabras, aquellas que nos permiten reflexionar a la vez que se produce la acción de proyectar, de este aspecto se desprende, casi naturalmente, la reflexión conjunta interactiva entre las partes, debemos interpretar aqueIlo que el estudiante nos comunica, a través de sus trabajos y sus palabras, para luego corregir los aspectos que todavía falta resolver y ponderar aquellos que están resueltos (Cutrera, 2016b, p. 5).

En este sentido destacamos que, en la ponderación de las resoluciones proyectuales, pertinentes a la idea planteada y a los objetivos pedagógicos, también se produce aprendizaje en el estudiante.

En los procesos de enseñanza y aprendizaje, docentes y estudiantes trabajamos sobre el proyecto expresado en dibujos, maquetas e incluso manifestado en la palabra escrita de las memorias proyectuales, sean estas conceptuales o descriptivas; en los talleres proyectuales se producen intercambios, generamos códigos que luego analizamos, para resignificar cada avance o cambio producido en el proyecto. En estos procesos utilizamos el diálogo y el lenguaje oral para manifestar pareceres, realizar correcciones y expresar críticas. Esta actitud dialógica colabora en la reflexión del estudiante sobre el proyecto y le permite accionar nuevamente sobre el proyecto y construir una nueva propuesta, la mayoría de 
las veces superior que la anterior como producto de estos procesos de intercambio.

En estas asignaturas, en la medida que los estudiantes aprenden a proyectar, los docentes les mostramos los modos en que se adquiere ese conocimiento durante el proceso proyectual. Entenderlos es primordial y fundacional para el crecimiento del estudiante como proyectista; generar en la comunicación del desarrollo del trabajo proyectual un discurso pautado, ordenado, sintético y claro resultará imprescindible para que el estudiante construya con el tiempo sus propios discursos con el lenguaje proyectual. "Partimos del concepto que a proyectar se aprende proyectando y que esta reflexión en la acción necesita de modos particulares de transmitir conceptos por parte del docente y asimilar conocimiento por parte del estudiante" (Cutrera, 2016b, p. 6). Cuando el estudiante comprende y aprende las palabras y los significados disciplinares ligados a ese lenguaje oral, está en condiciones de generar diálogos sobre elementos tangibles, como dibujos y maquetas, y sobre términos conceptuales propios de la disciplina y de la dinámica de la acción de proyectar, como intenciones, ideas o propuestas, que, previamente, solo están en los pensamientos del estudiante. A partir de nuestra experiencia como docentes, ligada al conocimiento de la temática y de los objetivos pedagógicos y disciplinares de los trabajos prácticos comprendemos el proyecto del estudiante, y como respuesta a este le brindamos alternativas que lo ayuden a avanzar en el proceso proyectual y de aprendizaje. Como docentes de talleres proyectuales, nuestros mensajes deben aludir a la acción de proyectar, pero sobre todo deben mostrar el proceso de aprendizaje que se desarrolla a la par del proceso de diseño, dicho de otro modo, tenemos el compromiso de evidenciar que mientras se proyecta se produce conocimiento.

Es muy frecuente, y sucede en casi la totalidad de las prácticas docentes de los talleres proyectuales, que el docente realice la corrección verbal asistida por dibujos y esquemas. En nuestra experiencia en los talleres de diseño, entendemos que acompañar el discurso con la acción de dibujar es una manera de reflexionar en la acción, nuestras palabras se complementan con los gráficos y se completan de significados. Comunicamos a nuestros estudiantes las alternativas posibles a seguir mediante dibujos, croquis y esquemas o bien operando sobre la modelización en maqueta del proyecto. Estas tácticas acompañan a nuestras palabras que ordenamos en un discurso coherente para brindar todos aquellos caminos posibles que pueda recorrer el estudiante para avanzar en el proceso proyectual, opciones que apuntan al aprendizaje de conceptos más que sus resultados concretos.

Algunos colegas docentes de asignaturas proyectuales sostienen que "dibujar soluciones" a los estudiantes no es pertinente. Afirman que estas acciones son modos de dar soluciones directas a problemas planteados por los proyectos, no permitiendo que el alumno busque la respuesta por sí solo. No obstante, consideramos que el estudiante usará esa información como referencia o posible alternativa a probar en el proceso de diseño, pudiendo resultar adecuada o no al desarrollo del proyecto. Además, es la palabra la que puede expresar diferentes soluciones representadas por esquemas parecidos, un dibujo casi abstracto puede representarnos poco, pero acompañado de determinado discurso puede ser una idea poderosa y pertinente a la situación dada.

Es el estudiante quien completa, plantea, proyecta y lleva el proceso de diseño, dependiendo siempre de sus ideas e intenciones; a su vez re interpreta los conceptos del docente, trata de comprenderlo y vuelve la mirada a su proyecto, está reflexionando en la acción (Cutrera, 2016 b, p. 6).

Esta ida y vuelta constituye una red de relaciones que se establece entre docente, estudiante y ejercicio proyectual. Como docentes explicamos en la acción sobre el proyecto 0 a través del dibujo conceptos intangibles pero fundamentales a la hora de aprender a proyectar (Cutrera, 2016b, p. 6).

Asimismo, en estas instancias, los docentes debemos estar dispuestos a escuchar, interpretar 
y comprender aquello que el estudiante está proyectando; una buena interpretación de sus intenciones nos dará pautas para guiarlo en posibles caminos a seguir en la toma de sus decisiones proyectuales, evaluando con sus propios criterios, aquellas alternativas que serán más adecuadas para dar respuesta a la problemática planteada.

Por otro lado, cuando los estudiantes interpretan una corrección y reflexionan sobre una crítica que realizan los docentes sobre los proyectos, comprenden que estas acciones generan aprendizajes en ellos y colaboran con el avance del proceso proyectual. Y luego, en el momento que ven los resultados de sus proyectos, reflexionan nuevamente, y reconocen una vez más que han aprendido.

\section{El silencio como espacio de reflexión}

Treinta radios convergen en el centro de una rueda, pero es su vacío lo que hace útil al carro. Se moldea la arcilla para hacer la vasija, pero de su vacío depende el uso de la vasija. Se abren puertas y ventanas en los muros de una casa, y es el vacío lo que permite habitarla. En el ser centramos nuestro interés, pero del no-ser depende la utilidad. (Libro del Tao. Verso 11 el vacío).

Estos versos, conformados por palabras concretas, nos comunican un mensaje abstracto. Las palabras las leemos, entendemos, podemos incluso estudiar sus etimologías, pero lo que nos trasmiten son conceptos. Y, a su vez, el concepto de estos versos nos muestra la importancia de lo ausente que finalmente hace a la esencia de cada cosa. Podríamos expresar que son tan importantes las materializaciones como las ausencias en lo que rezan los versos del Libro del Tao.

Recurrimos a esta cita para demostrar el valor de los silencios. Las palabras como conjuntos de signos cargadas de sentido no podrían ser tales si no existieran los espacios entre ellas, podríamos hacer una libre interpretación donde esos espacios son silencios que hacen a la esencia de la comunicación y a la comprensión de conceptos.
Ya hemos planteado algunas cuestiones vinculadas a la temática del lenguaje oral: cómo lo relacionamos con las disciplinas proyectuales, el rol que desempeña el diálogo entre los que participan del taller proyectual (docentes, estudiantes, proyectos), su importancia en los procesos de enseñanza y aprendizaje y en el proceso proyectual, entre algunas cuestiones.

Así nos referimos a diálogos, palabras, reflexiones y acciones propias de los procesos de aprendizaje que se dan en el marco de los procesos proyectuales. Nos centramos en el tema de las correcciones, las críticas y los vínculos que se establecen entre docentes y estudiantes (Cutrera, 2016b, p. 7).

Asimismo, entendemos que resulta significativo agregar a los conceptos desarrollados hasta aquí, otras cuestiones que nos parecen primordiales cuando reflexionamos sobre las distintas prácticas que se realizan en los talleres proyectuales. En primer término, señalamos la capacidad de observación que debe desarrollar el docente y que el estudiante debe ir aprehendiendo e incorporando en su proceso de aprendizaje. Como segunda cuestión consideramos importante respetar y valorar los silencios, "buscar detener la elaboración de juicios previos. (...) observar, permanecer en silencio dando lugar a la mirada, a lo que nos devuelve el observar" (Frigerio, Pescio y Piatelli, 2007, p. 45).

Una de nuestras tareas, quizás de las más significativas y difícil de lograr, como docentes de talleres proyectuales es escuchar al estudiante y entender qué intenciones tiene, cuáles son sus búsquedas y cuáles sus dudas, sin pre conceptos ni suposiciones previas, debemos guiarlo a aquello que él propone y no encausarlo hacia lo que nosotros pre suponemos; que nuestra mirada sea desprejuiciada, sin que por esto pierda rigor académico.

Si somos conscientes de la acción de mirar el proyecto del alumno desde este lugar podemos ayudarlo en su reflexión y en la construcción de su conocimiento. Por otro lado, ganaremos mayor objetividad sobre las cuestiones proyectuales y será más efectiva nuestra corrección o crítica, según el 
caso. Esto nos permite ser reflexivos, tener una actitud abierta para comprender (mejor las búsquedas y pensamientos de) nuestros estudiantes, ver con menos prejuicios las intenciones de los proyectos, dar lugar a las preguntas necesarias y permitirnos los silencios para escuchar al otro, (sea este) el estudiante, otro docente o el mismo proyecto (Cutrera, 2016b, p. 7).

Para que esta actitud de escucha, espera y disposición a la palabra del otro sea fructífera, y si estamos dispuestos a que se produzca diálogo, es imprescindible dar lugar al silencio. Debemos entrenarnos para generar esos espacios de silencios, brindarnos los docentes y estudiantes la posibilidad de escuchar las reflexiones que se producen cuando no tiene lugar la palabra en su expresión oral.

"En la música los silencios son parte de la partitura" (Cutrera, 2016b, p. 7). El músico necesita para componer tanto los sonidos como los silencios, nos resulta imposible imaginar una melodía sin sus pausas. Sin silencios no hay música. El arte de la música es un buen ejemplo para aprender de él, tan parecido e intangible como los espacios que generamos en la arquitectura. La comparación con la música nos permite valorar y comprender la importancia del silencio en los diálogos que se establecen en el proceso proyectual y en los procesos de enseñanza y aprendizaje del diseño; "distintos diálogos según sus protagonistas: entre los docentes y los estudiantes, entre los estudiantes y sus pares, el estudiante consigo mismo y entre el proyectista y el proyecto" (Cutrera, 2016b, p. 7), lo que llamamos el diálogo proyectual.

Así concluimos que la suma de los silencios y las palabras hacen al diálogo, construyen nuestras conversaciones. Los que participamos de los talleres de las asignaturas proyectuales, reflexionamos a partir de las preguntas que surgen de esos diálogos. Preguntamos y respondemos a los cuestionamientos que surgen del proceso proyectual en el proceso de enseñanza y aprendizaje. Pregunta el docente al estudiante 0 a otro docente, el estudiante al proyecto, a un par 0 a sí mismo; "y en las respuestas la palabra es mediadora, poética y académicamente necesaria" (Cutrera, 2016b, p. 7).

\section{Consideraciones sobre lo escrito. El texto culmi- na, las reflexiones no}

Aunque en las disciplinas proyectuales se utilizan lenguajes específicos como el dibujo, la modelización en maqueta entre otros, el lenguaje oral es el primer mediador para la comunicación entre los que participamos de las didácticas del taller proyectual, espacio en que los procesos pedagógicos se vinculan directamente con las prácticas reflexivas, donde el diálogo es una herramienta fundamental para fomentar el pensamiento proyectual.

Valoramos el diálogo entre los pares que conforman estas lógicas pedagógicas, estudiante/ docente, estudiante/estudiante, docente/docente, estudiante/proyecto y las interrelaciones que se producen en los procesos de enseñanza y de aprendizaje. Mediante ejercicios proyectuales los estudiantes proponen y proyectan sus intenciones, a través de los distintos tipos de diálogo que se dan en la dinámica del taller, se realizan correcciones, críticas y se generan debates que nos permiten "construir" conocimiento, fijar conceptos para luego trasladarlos nuevamente al objeto diseñado, constituyendo el proceso de diseño.

Por otro lado, consideramos sustancial para el proceso proyectual aprender y enseñar a escuchar y respetar los silencios. Tanto docentes como estudiantes debemos estar atentos a lo que expresa el otro y el proyecto mismo; en la conversación, proyectual y personal, tienen tanto peso las palabras y sus conceptos como los silencios que se generan. Así como el vacío hace a la esencia de un hecho arquitectónico, los silencios hacen a la construcción de los espacios de pensamiento.

Cada vez que abordamos un tema específico, indagamos solo algunos aspectos de la riqueza que conlleva la temática elegida. Quedan más dudas que certezas, apenas abrimos al gunos canales de investigación sobre la capacidad de la palabra como mediadora para aprehender conceptos concretos, sensoriales, intangibles de las disciplinas proyectuales.

Cada disciplina tiene un pensamiento y por lo tanto un lenguaje propio que deter- 
mina modos de expresión particulares. El proyectar y diseñar en arquitectura conllevan el desarrollo de un pensamiento y un lenguaje particular que denominamos lenguaje proyectual. El lenguaje es el límite y la expresión del pensamiento (Ben Altabef, 2018, p. 110).

Como docentes de talleres proyectuales debemos proponernos el objetivo de enriquecer el lenguaje de nuestros estudiantes; de todos los lenguajes que utilizamos en nuestras asignaturas, con el lenguaje oral iniciamos el vínculo entre docente y estudiante y a su vez el estudiante cierra su pro- ceso de aprendizaje con la expresión oral en cada ejercicio comunicando sus intenciones y resultados. Retomando la cita de Ben Altabef, si "el lenguaje es el límite y la expresión del pensamiento," entonces colaborar para que se "corra" lo más posible ese límite permitirá al estudiante enriquecer sus pensamientos y ganará herramientas para reflexionar sobre el proyecto en particular y, sobre todo, para formarse en la crítica y saberes de nuestras asignaturas proyectuales, para volver sobre el proyecto con más compromiso social y conocimiento disciplinar.

Notas

${ }^{1}$ Una versión preliminar y más breve de este artículo ha sido presentada en las III Jornadas De Investigadorxs, Grupos Y Proyectos De Investigación En Educación. Facultad de Humanidades/UNMdP. Junio 2016

${ }^{2}$ Cátedra Andrade. Introducción al Diseño Arquitectónico. FAUD/UNMdP. Argentina.

\section{Referencias bibliográficas}

Ben Altabef, C. (2018). "Intenciones para una didáctica proyectual. Caso: asignatura Proyecto y Forma en la FAU-UNT". En Cuaderno 67 Cuadernos del Centro de Estudios en Diseño y Comunicación, año 18, n 67 Buenos Aires UP.

Burbules, N. (1999). El diálogo en la enseñanza. Buenos Aires: Amorrortu.

Cutrera, C. (2016a). "La evaluación en las asignaturas proyectuales. La importancia del proceso proyectual en el aprendizaje". En Speranza, E., Calcagno, L. (Comp.) Reflexiones hacia una didáctica del proyecto....sobre el pensamiento proyectual y su práctica. Buenos Aires: UFLO.

Cutrera, C. (2016b). "Lenguajes de la disciplina proyectual. La palabra como mediadora". En II Jornadas de investigadorxs, grupos y proyectos de investigación en educación. Facultad de Humanidades/UNMdP. Mar del Plata. Obtenido de fh.mdp.edu.ar/encuentros/index.php/jie/3jie/paper/ download/1303/696

Frigerio, M. C., Pescio, S. y Piatelli, L. (2007). Acerca de la enseñanza del diseño. Reflexiones sobre una experiencia metodológica en la FADU. Buenos Aires: Nobuko.

Gregotti, V. (1972). El territorio de la arquitectura. Barcelona: Gustavo Gili.

Lao Zi (1990). El libro del Tao, Edición Bilingüe. Madrid: Alfaguara.

Mazzeo, Cy Romano A. M. (2007) La enseñanza de las disciplinas proyectuales. Hacia la construcción de una didáctica para la enseñanza superior. Buenos Aires: Nobuko.

Schön, D. (2010). La formación de profesionales reflexivos. Hacia un nuevo diseño de la enseñanza y el aprendizaje en las profesiones. Buenos Aires: Paidós. 